\title{
Meta Analysis of the Efficacy of Non- pharmaceutical Chinese Medicine Therapy in the Treatment of Obese Polycystic Ovary Syndrome
}

\author{
Shirong Liu ${ }^{1 *}$, Nan $\mathrm{Li}^{2}$ \\ 1Shaanxi University of Chinese Medicine, Xianyang 712000, Shaanxi Province, China \\ ${ }^{2}$ The Affiliated Hospital of Shaanxi University of Chinese Medicine, Xianyang 712000, Shaanxi Province, China \\ *Corresponding author: Nan Li, linan_80_311@163.com \\ Copyright: (C) 2022 Author(s). This is an open-access article distributed under the terms of the Creative Commons Attribution License (CC \\ BY 4.0), permitting distribution and reproduction in any medium, provided the original work is cited.
}

\begin{abstract}
Objective: To systematically evaluate the effects of acupuncture, auricular point sticking, acupoint catgut embedding, cupping and other non-pharmaceutical Chinese medicine therapies on sex hormone as well as glucose and lipid metabolism in patients with obese polycystic ovary syndrome. Method: The databases such as China National Knowledge Infrastructure were searched by computer to collect the literature on the treatment of obese polycystic by non-pharmaceutical Chinese medicine therapy combined with western medicine or lifestyle change, and the literature quality of the included literature was evaluated, and finally the data were analyzed. A total of 15 randomized controlled trial (RCT) literature were included in this study, with a total of 1263 patients. Results: Non-pharmaceutical Chinese medicine therapy combined with metformin or lifestyle can reduce body mass index, insulin resistance index, fasting plasma insulin, fasting blood glucose, luteinizing hormone, ratio of luteinizing hormone to follicle stimulating hormone, testosterone, but it has no obvious advantage over follicle stimulating hormone (FSH). Conclusion: Compared with simple application of metformin or lifestyle change, traditional Chinese medicine non drug therapy combined with metformin or lifestyle change can better improve sex hormone and blood glucose metabolism in obese PCOS patients.
\end{abstract}

Keywords: Obese polycystic ovary syndrome; Non-pharmaceutical Chinese medicine therapy sex hormone and blood glucose metabolism; Meta analysis

Online publication: January 19, 2022

\section{Introduction}

Polycystic ovary syndrome (PCOS) is a reproductive metabolic disease of women of childbearing age, including hyperandrogenism, ovulation disorder, fertility decline, obesity, insulin resistance and etc. Overweight is common in people with PCOS, ranging from 30 to $75 \%$. Obesity can cause insulin resistance or reduced glucose tolerance, both of which aggravate the symptoms of polycystic ovarian syndrome. Obese PCOS patients are being treated with first-line programs such as diet and exercise management, as well as western medication. However, it takes a long time to work, has a gradual impact, and long-term therapy has certain negative effects. Traditional Chinese medicine (TCM) has certain healing properties, but it also has the disadvantages of a long treatment cycle and delayed results. Acupuncture, moxibustion, cupping, acupoint catgut embedding, ear acupoint sticking, and other non-pharmaceutical Chinese medicine therapies are examples of TCM therapy. In order to find a safe and effective treatment for polycystic ovary syndrome patients, this paper aims to investigate the effects of non-drug therapy of TCM combined with 
western medicine or changing lifestyle on sex hormone, glucose, and lipid metabolism in obese polycystic ovary syndrome patients.

\section{Materials and methods}

\subsection{Inclusion criteria}

(1) The patient had clearly diagnosed obese polycystic ovary syndrome. Additionally, the diagnostic criteria of PCOS refer to the diagnostic criteria of PCOS recommended by European Society of Human Reproduction and Embryo (ESHRE) and American Society of Reproductive Medicine (ASRM) in Rotterdam Expert Meeting in 2003 or the criteria of PCOS promulgated and implemented by the Ministry of Health in 2011 . The obesity standard is BMI $\geq 25 \mathrm{~kg} / \mathrm{m}^{2}$, or waist circumference $\geq 80 \mathrm{~cm}$.

(2) The patients took one or more of acupuncture therapy, moxibustion, acupoint catgut embedding, massage and auricular point sticking, or combined western medicine or changed life style.

(3) The control group was treated with pure western medicine or lifestyle change, in which the drugs used in the experimental group and the control group must be the same in the same study.

(4) Outcome indicators included: Body mass index (BMI), glucose indexes (HOMA-IR, FINS, FBG), sex hormone. The included literature contained at least one of the observation indexes.

\subsection{Literature exclusion criteria}

The use of randomized control and other methods was not explained in the text. The material has been republished. Animal experiment, nursing, and review literature were among the topics covered in the literature. The study covered those with abnormal liver and renal function. Before and after self-control, the research literature was used. It is impossible to get reliable literature analysis data.

\subsection{Retrieval strategy}

CNKI, Wanfang Data Knowledge Service Platform, VIP database, PubMed and other databases were searched. The retrieval time limit is from the establishment of the database to November 1, 2020. "Acupuncture" or "auricular point sticking" or "acupoint catgut embedding" or "cupping" combined with "obese polycystic ovary syndrome" combined with "randomized control" were searched in Chinese.

\subsection{Literature screening and data extraction}

Two researchers independently browsed and screened the literature and extracted the data according to the inclusion criteria. The data extraction table mainly includes, basic information, research characteristics, intervention and control measures of the study. Research methodology and measurement data of outcome indicators.

\subsection{Risk assessment of bias included in the study}

Cochrane bias risk assessment tool was used to evaluate the methodological quality of the included literature. The quality evaluation results of each item can be divided into three levels, "low, high and uncertain bias risk."

\subsection{Statistical methods}

Revman5.3 software was used for statistical analysis. The clinical heterogeneity and statistical heterogeneity between studies were judged. Clinical heterogeneity was judged according to whether subjects, interventions, control measures and outcome indicators were similar in application details between studies, and statistical heterogeneity was judged according to the results of $\mathrm{I}^{2}$ test. 


\section{Results}

\subsection{Literature search results}

Based on this retrieval strategy, 276 potentially relevant articles were retrieved. After excluding unqualified literature, a total of 15 eligible studies were included in this meta-analysis.

\subsection{Characteristics of the included study}

There were a total of 1263 patients in 15 studies, including 645 cases in the experimental group and 618 instances in the control group. Acupuncture, acupoint catgut embedding, massage, auricular point sticking, and other non-pharmaceutical Chinese medicine treatments were used in the experimental group. The control group's intervention methods comprised western medicine therapy. Loss of follow-up or dropout was reported in the included literature ${ }^{[1-6]}$, but the rest was not described. Furthermore, the literature's outcome markers differ ${ }^{[7,8]}$ from those of the experimental group, which are master's theses. Observation documents based on theories ${ }^{[2]}$ are a $\mathrm{PhD}$ dissertation.

\subsection{Quality evaluation of the included study}

All the included literature mentioned randomized control, in which the data was ${ }^{[4-6,8-9,15]}$ used in the random number table, ${ }^{[2]}$ according to the order of treatment, ${ }^{[10]}$ grouped according to the computergenerated randomized control table ${ }^{[1]}$ used envelope method when assigning concealment, and mentioned sample size estimation and funding source. All the literature did not mention blind method, data integrity and whether there is selective reporting. The summary of bias risk of this meta-analysis is shown in Figure 1.

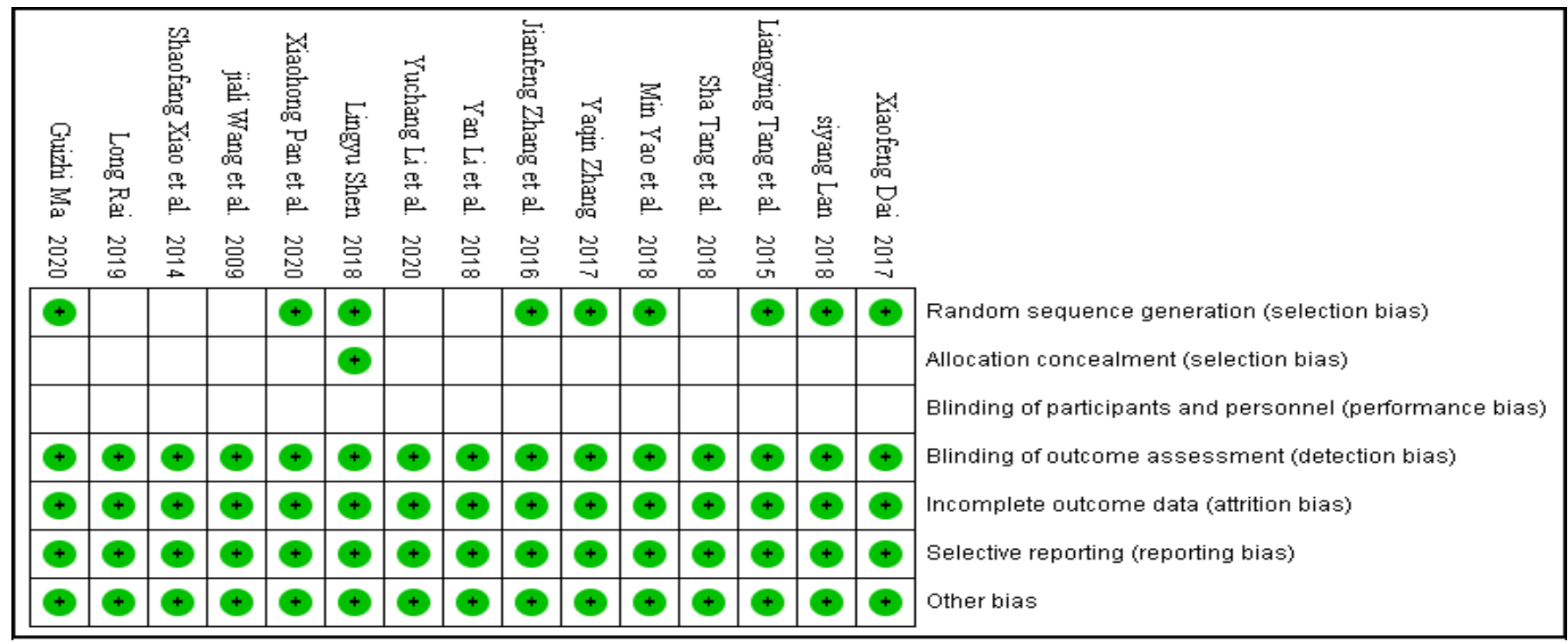

Figure 1. Summary chart of bias risk

\subsection{Meta analysis results}

\subsubsection{Body mass index}

There were 13 articles ${ }^{[1-12,14]}$ with BMI as the outcome index, including a total of 1093 subjects, $\mathrm{I}^{2}=91 \%>$ $50 \%, \mathrm{P}<0.01$, which had significant difference. The results were analyzed by random effect model $(\mathrm{MD}=$ $-1.52,95 \%$ CI $(-2.16,-0.87), \mathrm{P}<0.00001)$. The difference was statistically significant, suggesting that the effect of non-pharmaceutical Chinese medicine therapy combined with metformin or changing lifestyle to reduce $\mathrm{BMI}$ is better than that of metformin alone or changing lifestyle. 


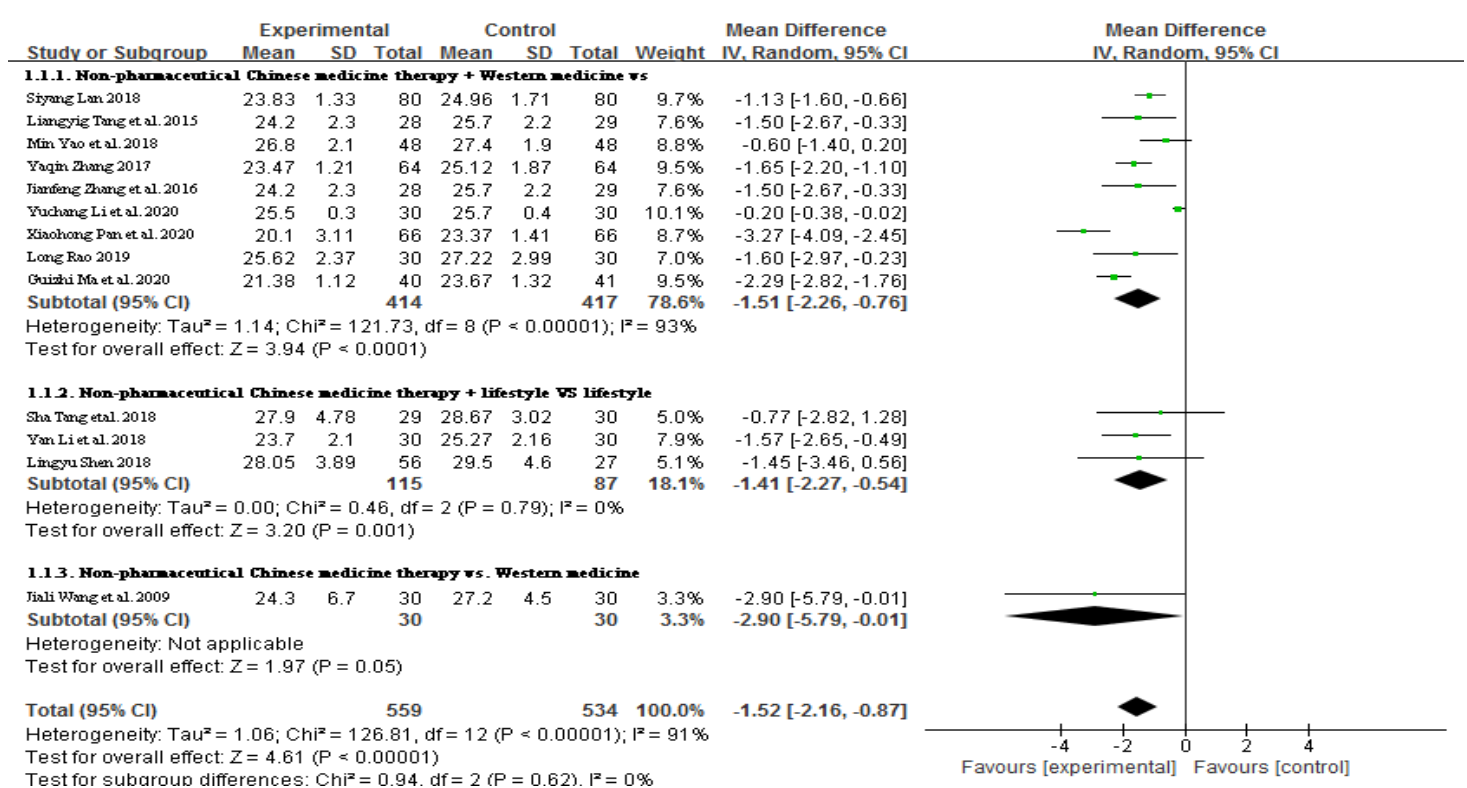

Figure 2. Results from random effect model

\subsubsection{Blood glucose}

There were 10 articles ${ }^{[1,2,5,7,9-13,15]}$ with FINS as the outcome index, including a total of 831 subjects, $\mathrm{I}^{2}=$ $93 \%>50 \%, \mathrm{P}<0.01$. There was heterogeneity. It was analyzed by random effect model $(\mathrm{MD}=-4.49,95 \%$ CI (-6.25, -2.74), $\mathrm{P}<0.00001)$, which has significant difference; There were 10 articles ${ }^{[2-5,7-13]}$ with FBG as the outcome index, including a total of 652 subjects, $\mathrm{I}^{2}=77 \%>50 \%, \mathrm{P}<0.01$. There was heterogeneity. It was analyzed by random effect model $(\mathrm{MD}=-0.18,95 \% \mathrm{CI}(-0.34,-0.01), \mathrm{P}=0.04<0.05)$, which has significant difference. There were 10 articles ${ }^{[1,2,4,5,9-13,15]}$ with HOMA-IR as the outcome index, including a total of 855 subjects, $\mathrm{I}^{2}=77 \%>50 \%, \mathrm{P}<0.01$. There was heterogeneity. It was analyzed by random effect model $(\mathrm{MD}=-0.46,95 \% \mathrm{CI}(-0.63,-0.29), \mathrm{P}<0.0001)$, which has significant difference. All the above suggest that the effect of non-pharmaceutical Chinese medicine therapy combined with metformin or changing lifestyle to reduce FINS, FBG and HOMA-IR is better than that of simple metformin or changing lifestyle.

\subsubsection{Sex hormone}

Table 1. The random effect model for sex hormones

\begin{tabular}{cccccccc}
\hline \multirow{2}{*}{$\begin{array}{c}\text { Sex } \\
\text { hormone }\end{array}$} & Inclusion study & $\begin{array}{c}\text { Total } \\
\text { number of } \\
\text { people }\end{array}$ & \multicolumn{2}{c}{$\begin{array}{c}\text { Heterogeneity test } \\
\text { results }\end{array}$} & \multicolumn{3}{c}{ Random effect model } \\
\cline { 5 - 8 } & & 818 & $92 \%$ & $<0.00001$ & 0.08 & $-0.47,0.64$ & 0.77 \\
\hline FSH & {$[2,4,6,11,13,15]$} & & & MD & 95\%cl & \multicolumn{2}{c}{ P } \\
LH & {$[2,4,6,10,11$,} & 946 & $89 \%$ & $<0.00001$ & -1.72 & $-2.57,-0.86$ & $<0.0001$ \\
LH/FSH & $12,4,6,10-13]$ & 788 & $87 \%$ & $<0.00001$ & -0.35 & $-0.60,-0.11$ & 0.005 \\
T & {$[2,4,6,8,10-13]$} & 788 & $95 \%$ & $<0.00001$ & -0.41 & $-0.74,-0.09$ & 0.01 \\
\hline
\end{tabular}

In the included literature, there was significant difference in the changes of LH, LH / FSH and T between the non-pharmaceutical Chinese medicine therapy combined with western medicine or lifestyle improvement group and the simple western medicine treatment or lifestyle improvement group $(\mathrm{P}<0.05)$. However, there was no significant difference in the changes of FSH $(\mathrm{P}>0.05)$.

Through meta-analysis, this study shows that non-pharmaceutical Chinese medicine therapy combined 
with western medicine or changing lifestyle has a higher therapeutic effect in the treatment of obese PCOS. Data analysis showed that the combination group had significant advantages in reducing BMI, blood glucose indexes (FINS, FBG, HOMA-IR) and sex hormones (LH, LH / FSH, T), and the difference was statistically significant. However, there may be no significant effect on some indexes, such as FSH, HDL$\mathrm{C}$ and WHR, and the difference is not statistically significant. It can be seen that compared with western medicine, non-pharmaceutical Chinese medicine therapy not only has significant therapeutic effect, but also has higher safety. Therefore, the effect of non-pharmaceutical Chinese medicine therapy combined with western medicine or changing lifestyle is better than that of simple western medicine or changing lifestyle.

This study also has some limitations, such as:

1. The hole taking is not fixed, the frequency is different, and the manufacturer and model of the buried line are not fixed.

2. Individual physical differences lead to different sensitivity or tolerance to non-pharmaceutical treatment.

3. The difference of obesity diagnostic criteria may lead to the difference of outcome indicators, and ultimately affect the results of meta-analysis.

4. The difference of sex hormone, blood glucose and insulin detection methods and kits may also be one of the factors affecting the research results.

The quality of the literature included in this study is low. All the literatures do not mention the blind method, data integrity and the existence of selective reports, which will also reduce the authenticity of the research results.

In conclusion, non-pharmaceutical Chinese medicine therapy has a certain therapeutic effect on obese PCOS patients. Non-pharmaceutical Chinese medicine therapy combined with western medicine or lifestyle adjustment therapy can effectively reduce BMI, blood glucose, insulin (FINS, FBG, HOMA-IR) and sex hormones (LH, LH / FSH, T), but its specific mechanism needs to be further discussed.

\section{Disclosure statement}

The author declares no conflict of interest.

\section{References}

[1] Ma G, Hu Z, Shi Y, et al., 2020, Effect of Acupoint Catgut Embedding Combined with Metformin on Glucose and Lipid Metabolism in Obese Patients with Polycystic Ovary Syndrome. Shanghai Journal of Acupuncture and Moxibustion, 39(09): 1123-1127.

[2] Shen L, 2018, Observation on the Curative Effect of Treating Abdominal Obese Polycystic Ovary Syndrome from the Theory of Pulse Taking and Exploration of Kisspeptin Related Mechanism. Zhejiang Chinese Medical University.

[3] Tang S, Feng X, Xu D, et al., 2018, Clinical Observation of Acupoint Catgut Embedding Combined with Cupping Therapy on Body Mass of Obese Patients with Polycystic Ovary Syndrome. Guangming Journal of Chinese Medicine, 33(18): 2710-2711.

[4] Yao M, Ding D, Zhou W, et al., 2018, Effect of Acupuncture and Moxibustion on Anxiety in Obese Patients with Polycystic Ovary Syndrome. ACTA Chinese Medicine, 33(10): 2043-2048.

[5] Zhang J, Huang R, Tang L, et al., 2016, Effect of Acupoint Catgut Embedding on Blood Glucose and Insulin Indexes in Patients with PCOS. Guangming Journal of Chinese Medicine, 31(06): 831-833.

[6] Tang L, Huang R, Zhang J, 2015, Effect of Acupoint Catgut Embedding on Sex Hormone Related Indexes in Obese Patients with Polycystic Ovary Syndrome. Clinical Journal of Chinese Medicine, 
7(21): 60-62.

[7] Rao L, 2019, Clinical Study on the Effect of Electroacupuncture Combined with Cupping on Pituitary Hormone and Glucose and Lipid Metabolism in Obese PCOS Patients. Shaanxi University of Chinese Medicine.

[8] Lan C, 2018, Observation on the Efficacy of Acupuncture and Auricular Point Pressing Combined with Metformin in the Treatment of Obese Polycystic Ovary Syndrome. Hebei Medical University.

[9] Li Y, Feng T, He M, 2020, Effect of Auricular Bean Pressing Combined with Metformin on Insulin Resistance in Obese Patients with Polycystic Ovary Syndrome. Chinese Medical Innovations, 17(25): 153-157.

[10] Zhang Y, 2017, Effects of Acupuncture and Moxibustion on Adiponectin, Leptin and Insulin Resistance in Patients with Obese Polycystic Ovary Syndrome. ACTA Chinese Medicine, 32(11): 2259-2262.

[11] Pan X, Sun P, Qin W, et al., 2020, Clinical Study on Acupoint Catgut Embedding Combined with Thunder Fire Moxibustion in the Treatment of Obese Polycystic Ovary Syndrome. Journal of Practical Traditional Chinese Medicine, 36(11): 1406-1408.

[12] Li Y, Hao S, Zhang C, et al., 2018, Clinical Observation of Ear Point Seed Pressing in the Treatment of Adolescent Overweight / Obese Polycystic Ovary Syndrome. Modern Journal of Integrated Traditional Chinese and Western Medicine, 27(35): 3877-3879 + 3906.

[13] Xiao S, Xu Y, Zhu L, et al., 2014, Acupoint Catgut Embedding Combined with Diane and Metformin in the Treatment of 36 Cases of Obese Polycystic Ovary Syndrome. Jiangxi Journal of Traditional Chinese Medicine, 45(04): 53-55.

[14] Wang J, Zhang Z, 2009, Acupuncture Combined with Acupoint Catgut Embedding in the Treatment of 30 Cases of Obese Polycystic Ovary Syndrome. China Association of Chinese Medicine. Proceedings of the Ninth National Conference of TCM Gynecology. China Association of Chinese Medicine: Chinese Society of Traditional Chinese Medicine, 3.

[15] Dai X, 2017, Clinical Observation of Acupuncture, Auricular Point Sticking and Pressure Combined with Metformin in the Treatment of Obese Polycystic Ovary Syndrome Infertility. Journal of New Chinese Medicine, 49(04): 128-131. 